\title{
Transient Stability Analysis of Power System with Photovoltaic Systems Installed
}

\author{
Masaki Yagami ${ }^{1}$, Seiichiro Ishikawa ${ }^{1}$, Yoshihiro Ichinohe ${ }^{1}$, Kenji Misawa ${ }^{1}$ and Junji Tamura ${ }^{2}$ \\ 1. Department of Electrical and Electronic Engineering, Hokkaido University of Science, Sapporo 006-8585, Japan \\ 2. Department of Electrical and Electronic Engineering, Kitami Institute of Technology, Kitami 090-8507, Japan
}

Received: August 27, 2015 / Accepted: September 14, 2015 / Published: October 31, 2015.

\begin{abstract}
The impact of large-scale grid-connected PV (photovoltaics) on power system transient stability is discussed in this paper. In response to an increase of PV capacity, the capacity of conventional synchronous generator needs to be reduced relatively. This leads to the lower system inertia and the higher generator reactance, and hence, the power system transient stability may negatively be affected. In particular, the impact on the transient stability may become more serious when the considerable amounts of PV systems are disconnected simultaneously during voltage sag. In this work, the potential impact of significant PV penetration on the transient stability is assessed by a numerical simulation using PSCAD/EMTDC.
\end{abstract}

Key words: Photovoltaic, LVRT (low-voltage ride-through), synchronous generator, infinite bus, transient stability.

\section{Introduction}

With the increasing concerns about global warming, the amount of solar PV (photovoltaic) installations is growing worldwide. In particular, in Japan, the investment in the field of PV power generation has been increasing after the Fukushima nuclear power plant accident. During 2013, a total of $6.9 \mathrm{GW}$ of PV was installed in Japan, $400 \%$ increase beyond the installation in the previous year $(1.7 \mathrm{GW})$. Cumulative installed capacity of PV systems in 2013 reached 13.6 GW [1]. Japan introduced a feed-in tariff scheme for renewable energy in July, 2012. The PV market, therefore, is expected to continue growing significantly. However, the expanding installation of PV has a significant impact on the power system behavior.

With the increasing of PV capacity in the power system, several thermal power plants need to be closed down or at least the output power of thermal unit must be decreased in order to maintain power

Corresponding author: Masaki Yagami, Ph.D., associate professor, research fields: power system stability and FACTS (flexible AC transmission system). E-mail: yagami@hus.ac.jp. supply-demand balance. However, as the generator operation with low output leads to an increase in operating costs, reduction of the generator capacity would mainly be taken. As a result, the transient stability would negatively be affected due to the lower system inertia and the higher generator reactance. On the other hand, when a fault occurs in the power system, some parts of PV systems may be disconnected from the grid due to the voltage sag. The disconnection causes a drop in PV generation, and hence a further voltage drop in the grid system may be induced. Eventually, the considerable amounts of PV systems may be disconnected from the grid. In this case, not only the transient stability would be affected, but also the frequency stability would significantly be affected due to the large power imbalance and the fewer frequency control generators. In order to prevent the frequency drop, power system operators would require PV systems to be equipped with LVRT (low-voltage ride through) capability. LVRT requirements stipulate that $\mathrm{PV}$ needs to stay connected during a temporary fault to provide post-fault voltage support [2]. 
In this paper, the impact of large-scale grid-connected PV with or without LVRT capability on the power system transient stability is discussed based on the numerical simulation analysis using PSCAD/EMTDC software. The salient feature of this work is that, the transient stability analysis is carried out for two power system models with and without infinite bus respectively. Namely, PV systems connected with a large power system with an infinite bus or a comparatively small system without an infinite bus are considered in the transient stability simulation. In the near future, the disconnection of GW-scale PV system will be occurred. In large power system, the influence of loss of active power from PV on the transient stability may be small due to the sufficient power supply from other power source including infinite bus. On the other hand, in small power system, the synchronous generator may be subjected to large decelerating power when PV is disconnected, and hence, in extreme cases, the generators may go to out-of-step in decelerating direction.
Although there are some studies relating to the stability analysis using the power system model with infinite bus and PV system [3-5], few studies has focused on the influence of infinite bus on the transient stability. As the transient stability is mainly an instantaneous energy balance problem, it is important to consider the influence of the existence of LVRT capability and infinite bus on the transient stability.

The paper is organized as follows: Section 2 describes the simulation model; Section 3 presents the simulation results and discussions; Section 4 gives conclusions.

\section{Simulation Model}

\subsection{Power System Model}

Fig. 1 shows 9-bus power system model [6] used in the simulation analysis, which is composed of three power sources, one synchronous generator of $500 \mathrm{MVA}$ (SG1), an infinite bus or another synchronous generator of 500 MVA (SG2), and a large-scale PV plant of $500 \mathrm{MW}(\mathrm{PV})$ or a third synchronous

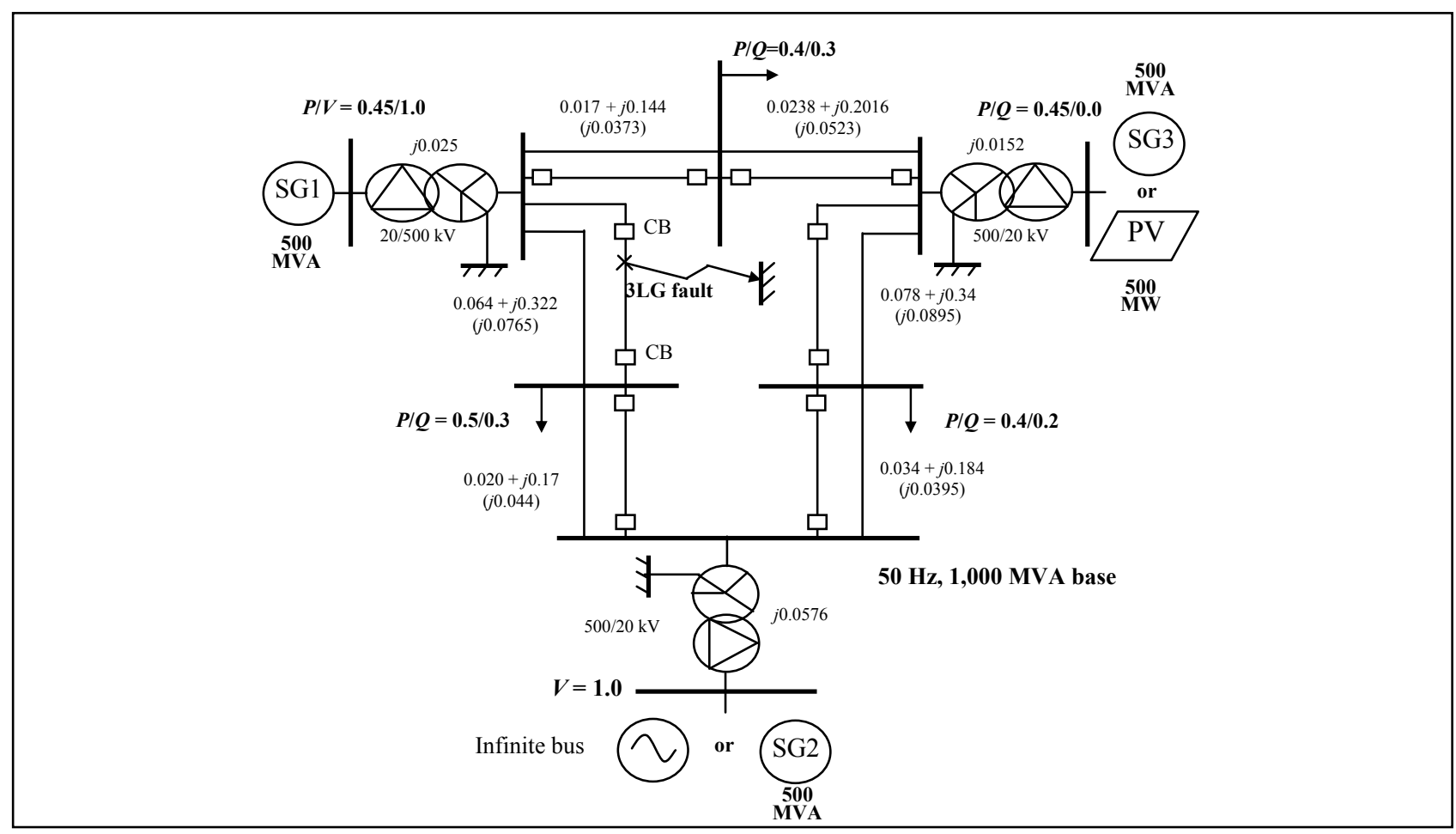

Fig. 1 Power system model. 
Table 1 Synchronous generator parameters.

\begin{tabular}{llllll}
\hline & \multicolumn{3}{c}{ Generator parameters } \\
\hline & SG1 & SG2 & & SG1 & SG2 \\
\hline$R_{a}(\mathrm{pu})$ & 0.003 & 0.004 & $X_{q}{ }^{\prime}(\mathrm{pu})$ & 0.171 & 0.134 \\
$X_{l}(\mathrm{pu})$ & 0.102 & 0.078 & $T_{d o}{ }^{\prime}(\mathrm{s})$ & 5.9 & 8.97 \\
$X_{d}(\mathrm{pu})$ & 1.651 & 1.22 & $T_{q o}{ }^{\prime}(\mathrm{s})$ & 0.535 & 1.5 \\
$X_{q}(\mathrm{pu})$ & 1.59 & 1.16 & $T_{d o}{ }^{\prime}(\mathrm{s})$ & 0.033 & 0.033 \\
$X_{d}{ }^{\prime}(\mathrm{pu})$ & 0.232 & 0.174 & $T_{q o}{ }^{\prime}(\mathrm{s})$ & 0.078 & 0.141 \\
$X_{q}{ }^{\prime}(\mathrm{pu})$ & 0.38 & 0.25 & $H(\mathrm{~s})$ & 3.0 & 3.0 \\
$X_{d}{ }^{\prime}(\mathrm{pu})$ & 0.171 & 0.134 & & & \\
\hline
\end{tabular}

generator of 500 MVA (SG3). Each power source is connected through step up transformer to $500 \mathrm{kV}$ bus and double circuit transmission lines. The impedances and the initial power flows are shown in per unit values on the power system base of 1,000 MVA in Fig. 1. In the case with PV installed, the existence of LVRT capability is considered for PV inverter. LVRT characteristic considered in this work is simple. The PV inverter with LVRT capability maintains the connection to the grid even if its terminal voltage is dropped. On the other hand, the PV inverter without LVRT capability is disconnected from the grid when its terminal voltage drops below $70 \%$ of the nominal voltage [7], and the disconnection is maintained for remaining period of the simulation.

The parameters used for each synchronous generator are shown in Table 1. AVR (automatic voltage regulator) [8] and governor control systems shown in Fig. 2 have also been included in each generator model.

In the power system model, symmetrical 3LG (three-line-to-ground) fault is assumed as network disturbance. The fault occurs near SG1 at $0.1 \mathrm{~s}$, the CBs (circuit breakers) on the faulted lines are opened at $0.17 \mathrm{~s}$, and at $1.17 \mathrm{~s}$, they are reclosed. For $500 \mathrm{kV}$ transmission lines in Japan, reclosing with a fixed time interval of 50 cycles (i.e., $1.0 \mathrm{~s}$ for $50 \mathrm{~Hz}$ system) has been adopted.

\subsection{PV System Model}

The PV system model is shown in Fig. 3. It consists of PV module, inverter and LC (low-pass) filter. The

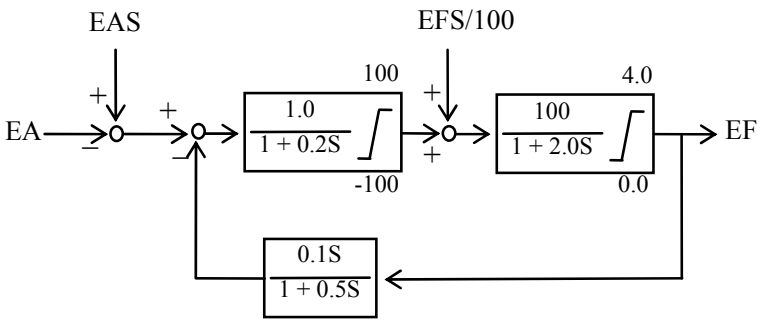

EA: terminal voltage; EAS: terminal voltage reference; EF: field voltage; EFS: field voltage reference

(a) AVR

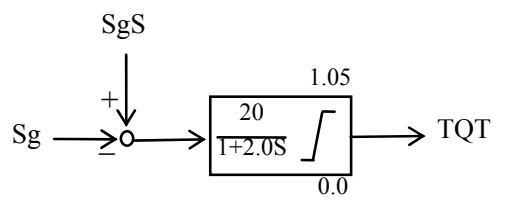

Sg: generator speed SgS: generator speed reference TQT: turbine torque

(b) Governor

Fig. 2 AVR and governor models.

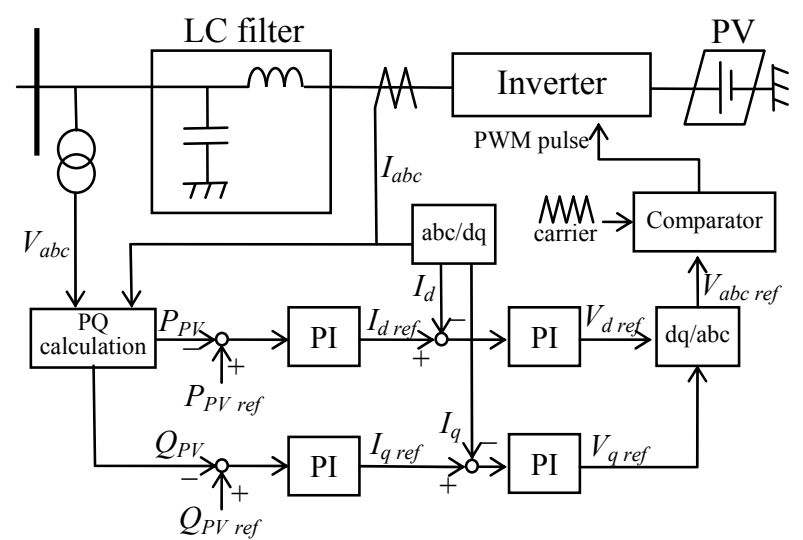

Fig. 3 Control block diagram of PV inverter.

PV module is represented with a voltage source, and hence the PV output is constant under the steady state. This means irradiation and PV cell temperature are 
constant during the simulation period. In general, transient stability is analyzed in a time window of a few seconds to several tens of seconds. The assumption, therefore, may be valid for the transient stability analyses. The genetic PWM (pulse-width modulation) voltage source converter is used as PV inverter. The carrier frequency is $10 \mathrm{kHz}$. The inverter controls the active and reactive power injected from the PV module to the system. To maintain the active and reactive power at the reference set points, the currents of the inverter are controlled by using vector control technique. The current limitation for over current is not considered in this model. Currently, most of the $\mathrm{PV}$ inverters are designed to operate at unity power factor [7], and therefore the reference value of the reactive power is set to zero in each case.

\section{Simulation Results}

\subsection{Stability of Power System with Infinite Bus}

First, the transient stability of the power system with infinite bus is discussed. Fig. 4 shows the phase angle responses of SG1. To compare the effects of the conventional synchronous generator and PV system on transient stability, we have considered three simulation conditions in terms of the power sources as follows: (1) connecting SG3 instead of PV, (2) connecting PV without LVRT capability, and (3) connecting PV with LVRT capability. As can be seen, the first peak of the phase angle swing is decreased in the case of PV without LVRT. In other words, risk of out-of-step of the synchronous generator becomes low in the power system installed with PV without LVRT.

Fig. 5 shows the kinetic energy responses of SG1. As can be seen, in the case with PV without LVRT implemented, the kinetic energy stored in the rotor of SG1 can be released quickly compared with other cases. PV without LVRT is disconnected from the grid due to the voltage sag when the fault occurs. The disconnection of PV increases the power load of SG1, and hence the output power of SG1 is enlarged. On the other hand, as the prime-mover mechanical power

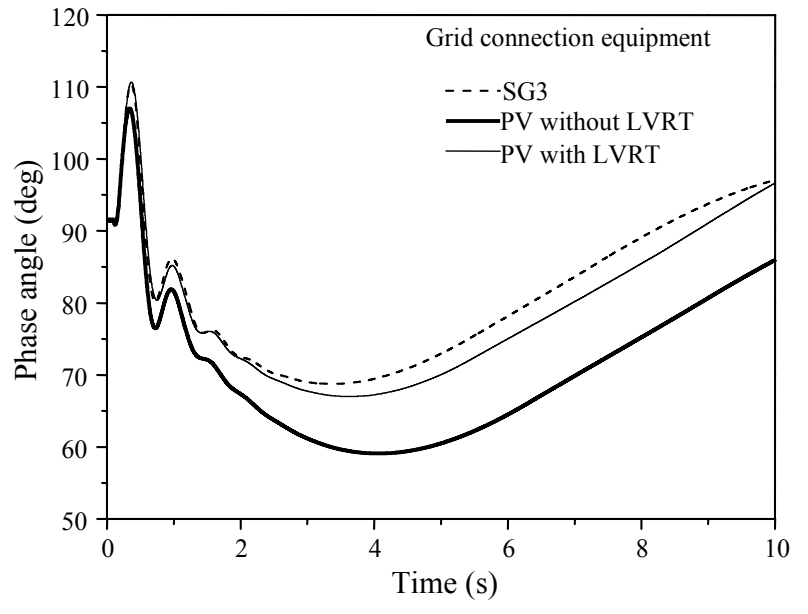

Fig. 4 Phase angle of SG1 (with infinite bus).

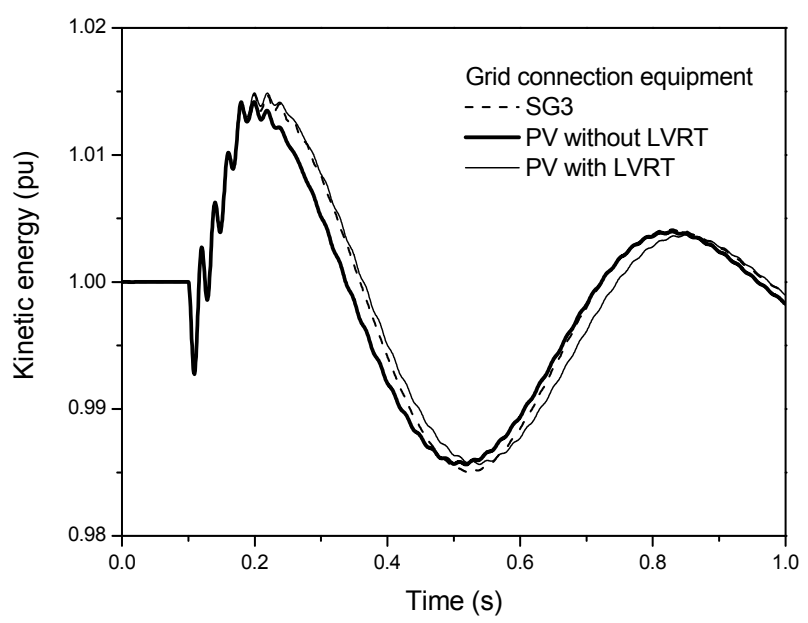

Fig. 5 Kinetic energy of SG1 (with infinite bus).

of SG1 cannot be increased quickly, the output power of SG1 is supplied from the kinetic energy which is stored during the period of acceleration. The kinetic energy stored in the rotor is released quickly, and hence the swings of the phase angle and the kinetic energy are restrained in the case of PV without LVRT.

Fig. 6 shows the active power of each power source in the case with PV installed with or without LVRT implemented. In both cases, when the fault occurs, active powers of SG1 and PV decrease due to the voltage sag. On the other hand, the active power from infinite bus increases during the period of fault because the bus voltage is a constant. After the fault clearing, the active power of SG1 increases because the terminal voltage of SG1 is recovered. In the case with PV with LVRT implemented, the active power of 


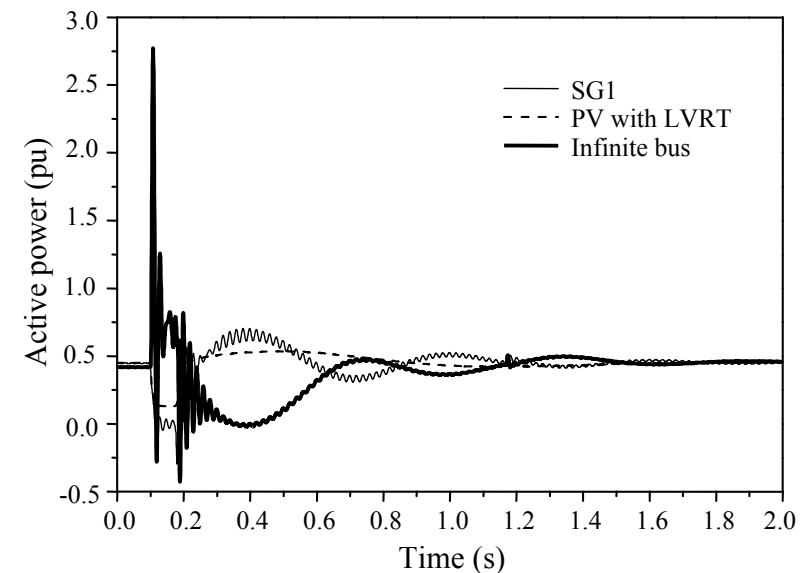

(a) PV with LVRT

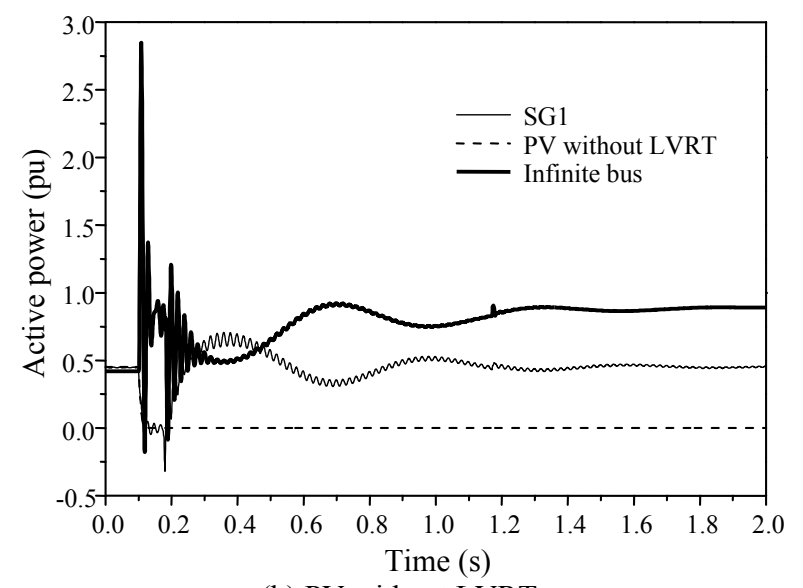

(b) PV without LVRT

Fig. 6 Active powers from SG1, PV and infinite bus in the case with PV with/without LVRT implemented (with infinite bus).

$\mathrm{PV}$ is also increased. To maintain power supply-demand balance, therefore, the active power from the infinite bus does not increase so much. The active power of each power source converges so as to approach to the value in steady state. On the other hand, in the case with PV without LVRT implemented, the active power of $\mathrm{PV}$ is maintained at $0 \mathrm{pu}$ because PV without LVRT is disconnected from the grid due to the voltage sag. The power shortage caused by the disconnection of PV is covered by active powers of SG1 and infinite bus. However, as maximum value of output power of SG1 is restricted by a turbine load limiter, the active power from infinite bus gets extremely large. In small power system, the power shortage must only be covered by the active powers of power sources in the local network.

\subsection{Stability of Power System without Infinite Bus}

Next, the transient stability of the power system without infinite bus is discussed. The synchronous generator SG2 is connected with the grid instead of infinite bus. The initial power flow is the same with that of the case with infinite bus connected.

Fig. 7 shows the phase angle responses of SG1 and SG2. The phase angles shown are displacement angle with respect to the phase angle of the swing generator $\mathrm{SG} 2$. As can be seen, the first peak of the phase angle swing of SG1 in the case with PV with LVRT implemented is decreased more than those in other cases. On the other hand, the phase angle in the case with PV without LVRT implemented increases significantly, and then decreases toward 0 degrees. Fig. 8 shows the kinetic energy responses of each synchronous generator. As can be seen, in the case with PV without LVRT implemented, the kinetic energies of SG1 and SG2 decrease toward $0 \mathrm{pu}$. Namely, SG1 and SG2 go to out-of-step in the decelerating direction.

Figs. 9-11 show the responses of the active power, the reactive power, and the terminal voltage of each power source respectively. As can be seen in Figs. 9a and $9 \mathrm{~b}$, in the case with PV without LVRT implemented, the active powers of SG1 and SG2 rise up significantly after the fault clearing at $0.17 \mathrm{~s}$. This is because to maintain the power supply-demand

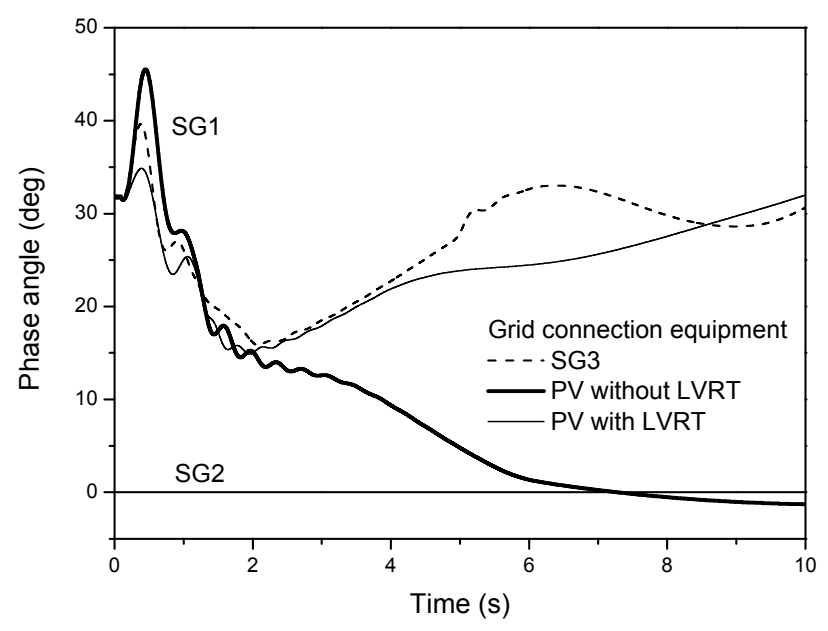

Fig. 7 Phase angles of SG1 and SG2 (without infinite bus). 


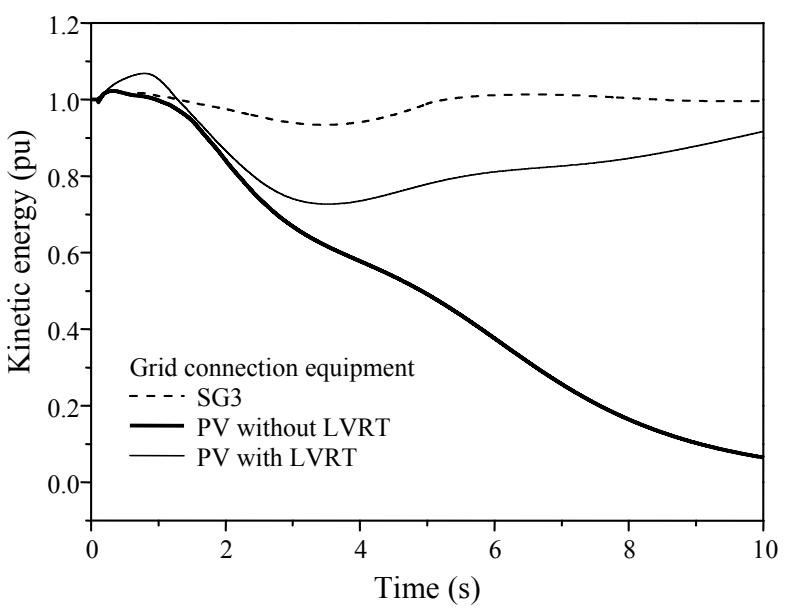

(a) SG1

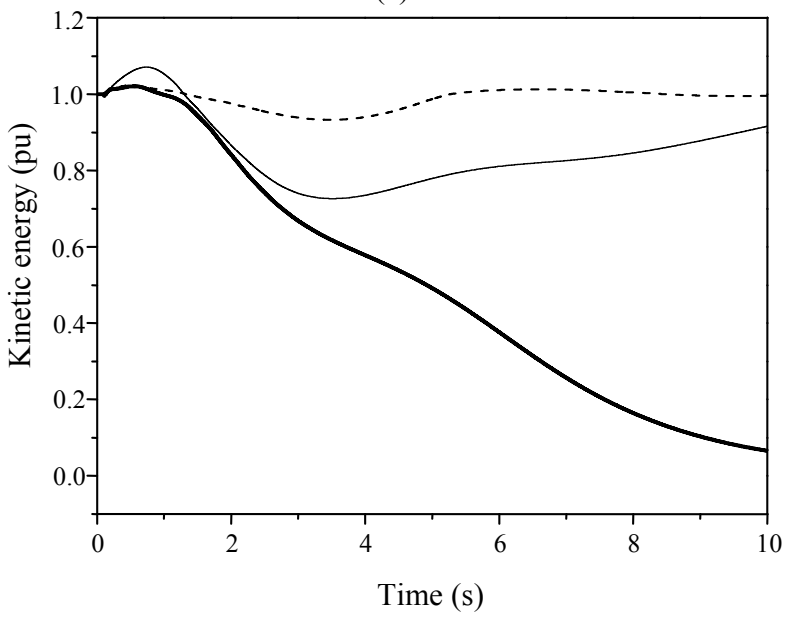

(b) SG2

Fig. 8 Kinetic energies of SG1 and SG2 (without infinite bus).

balance after the disconnection of PV. However, as the prime-mover mechanical power of synchronous generator is restricted by the turbine load limiter, the rotor is subjected to a large decelerating power due to unbalance between the mechanical input power and the electrical output power. As a result, the kinetic energy stored in the rotor is extremely released, and hence the generator goes to out-of-step in the decelerating direction.

In the case of PV with LVRT, the active powers of SG1 and SG2 also rise up significantly after the fault clearing because the active power of PV is temporarily decreased at around $1.5 \mathrm{~s}$. However, SG1 and SG2 do not go out-of-step because the power supply from PV is maintained after the fault.
PV inverter is controlled to maintain both active and reactive power at the reference set points. As a result, the large current flows from PV to the grid when the terminal voltage drops due to the fault. Therefore, as seen in Fig. 9c, the active power from PV becomes very large when the terminal voltage of $\mathrm{PV}$ is recovered by clearing the fault. In addition, as the reactive power from PV with LVRT increases around $1.0 \mathrm{~s}$ as seen in Fig 10c, the terminal voltage of $\mathrm{PV}$ also increases as seen in Fig. 11c. As a result, the active power from PV increases significantly around this time. To maintain the power supply-demand balance, the active powers of SG1 and SG2 after the fault clearing is maintained at low values until about $1.0 \mathrm{~s}$. As a result, a large amount of kinetic energy is stored in the rotor, and hence the synchronous generators of SG1 and SG2 accelerate more than those of the case with SG3 installed.

As can be seen in Fig. 8, in the case of PV with LVRT implemented, the kinetic energies of SG1 and SG2 rise up significantly after the fault. As mentioned earlier, this is because the generator output after the fault clearing cannot be enlarged due to the active power from PV with LVRT. As seen in Fig. 7, the phase angle swing of SG1 in the case with PV with LVRT implemented is restrained significantly. Namely, the risk of out-of-step of the synchronous generator becomes low in the power system with PV with LVRT implemented. However, acceleration or deceleration of the rotor causes severe mechanical stresses. Therefore, in order to release quickly the kinetic energy stored in the rotor, it might be worth changing the control mode of PV inverter to constant voltage control. If PV supplies a sufficient reactive power after the fault, the terminal voltage and the active power of the synchronous generator increase. Therefore, the synchronous generator may be subjected to the decelerating power due to unbalance between the mechanical input power and the electrical output power, and the kinetic energy deviation may be restrained. 


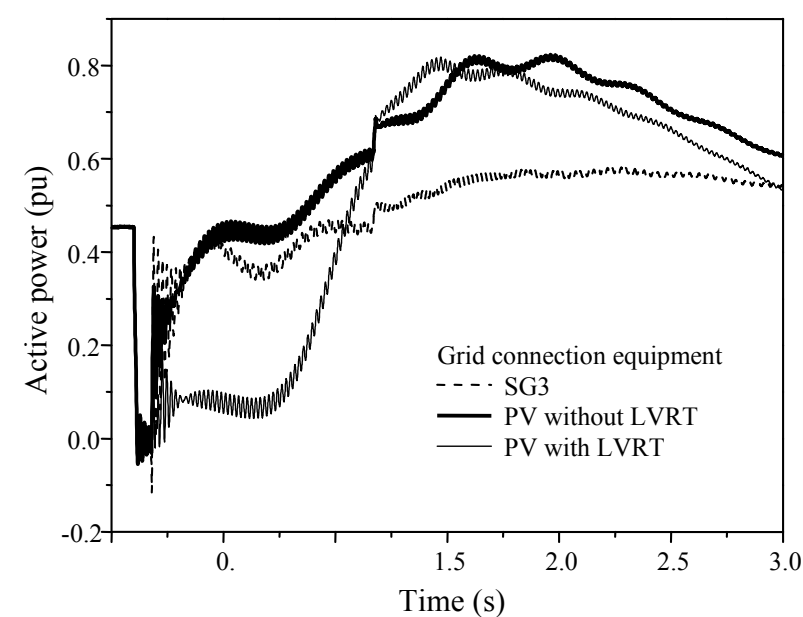

(a) SG1

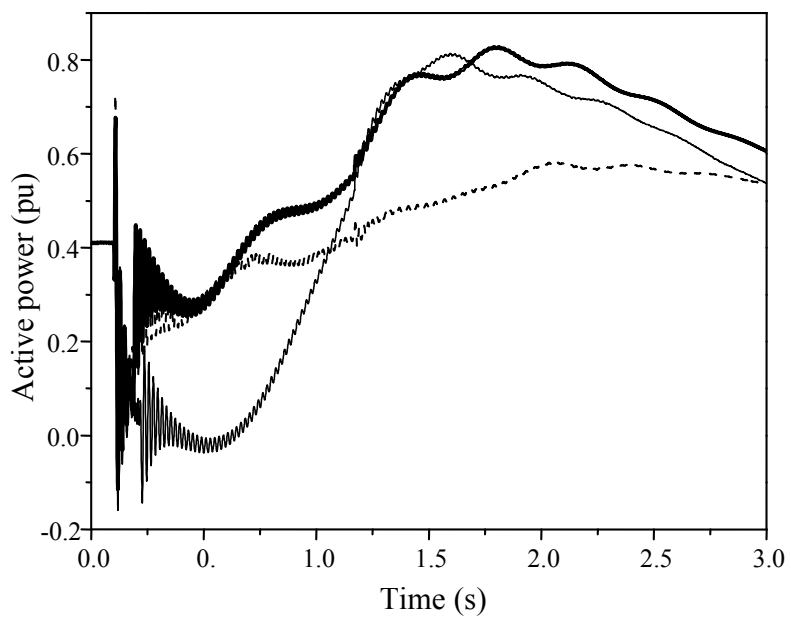

(b) SG2

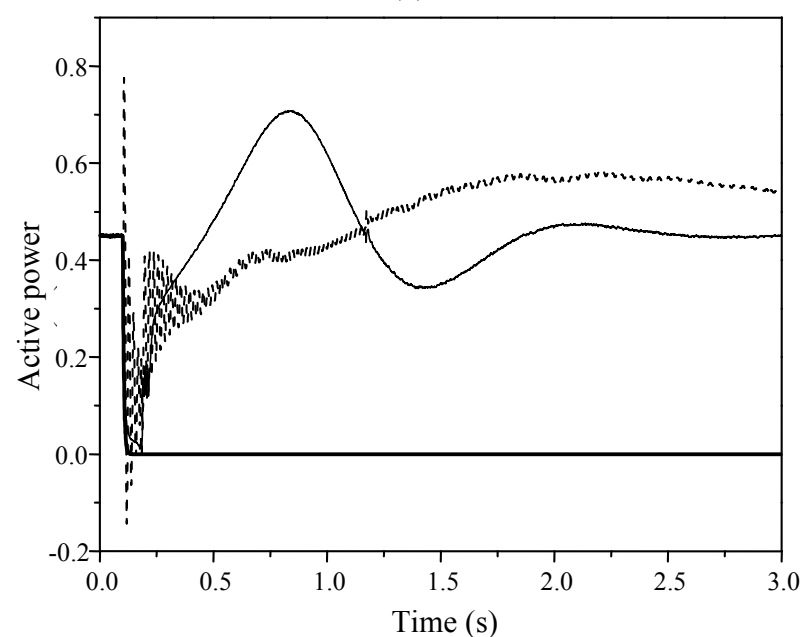

(c) PV or SG3

Fig. 9 Active powers from SG1, SG2 and PV or SG3 (without infinite bus).

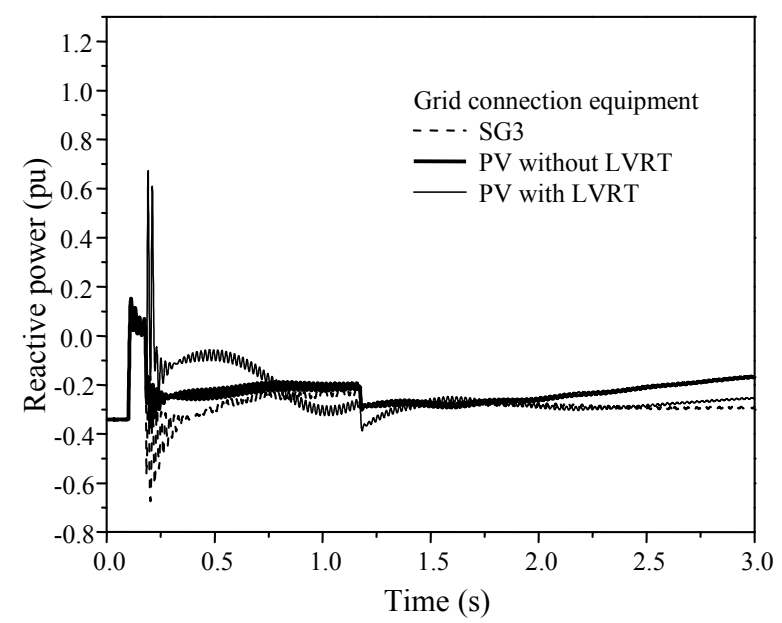

(a) SG1

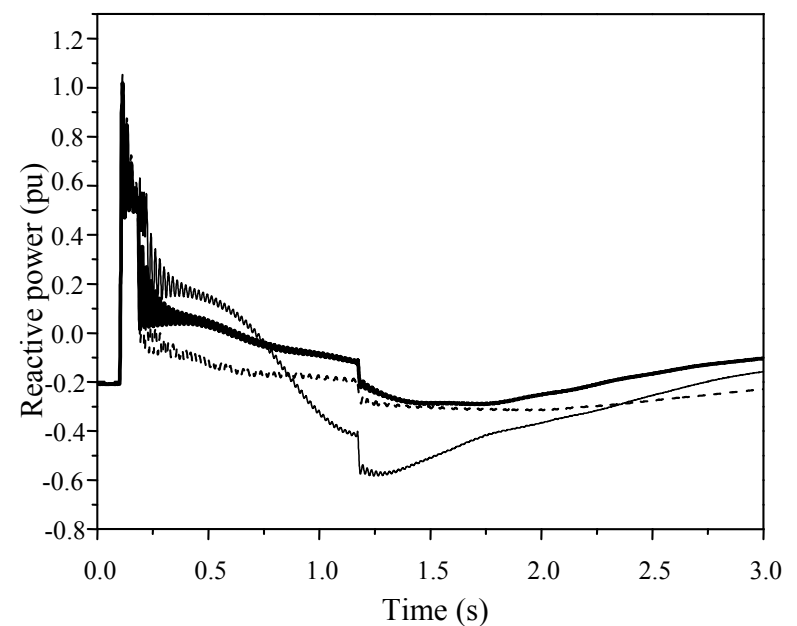

(b) SG2

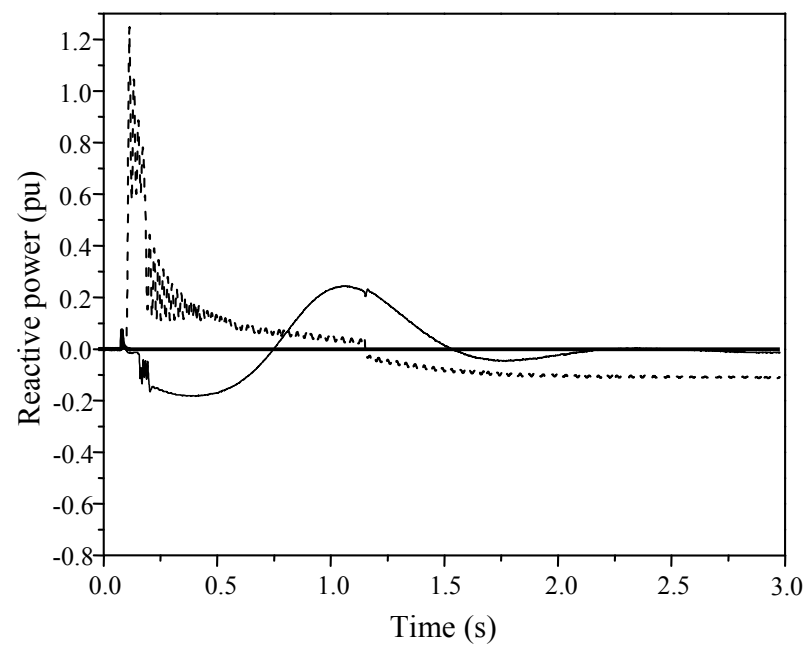

(c) PV or SG3

Fig. 10 Reactive powers of SG1, SG2 and PV or SG3 (without infinite bus). 


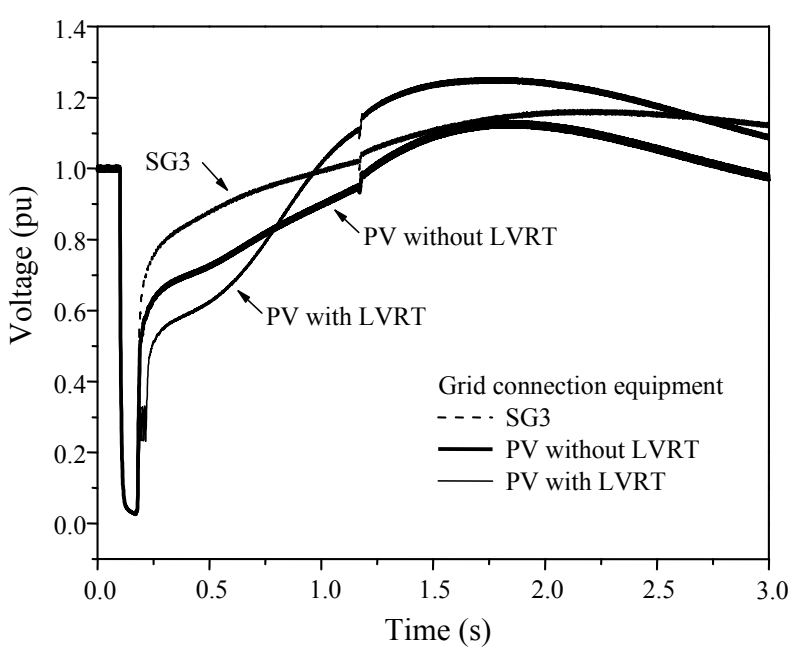

(a) SG1

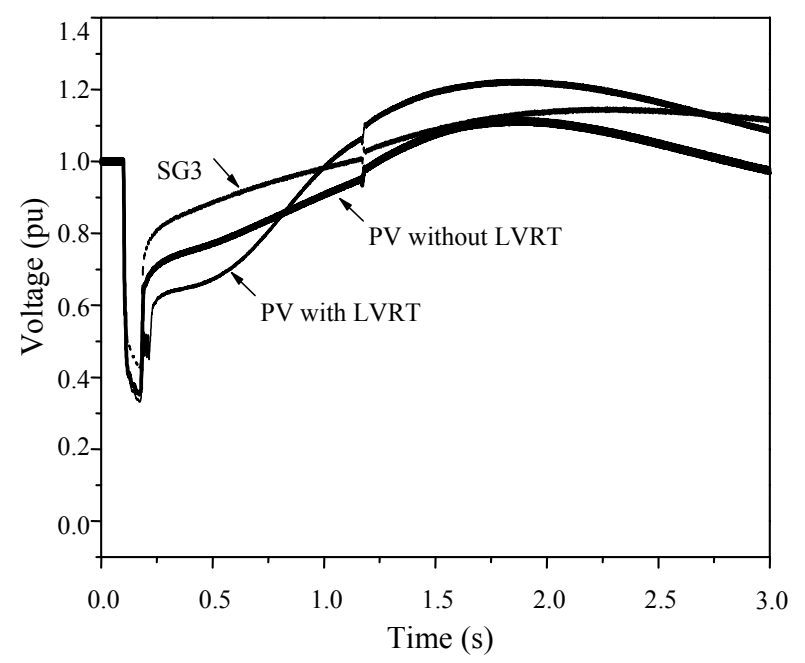

(b) SG2

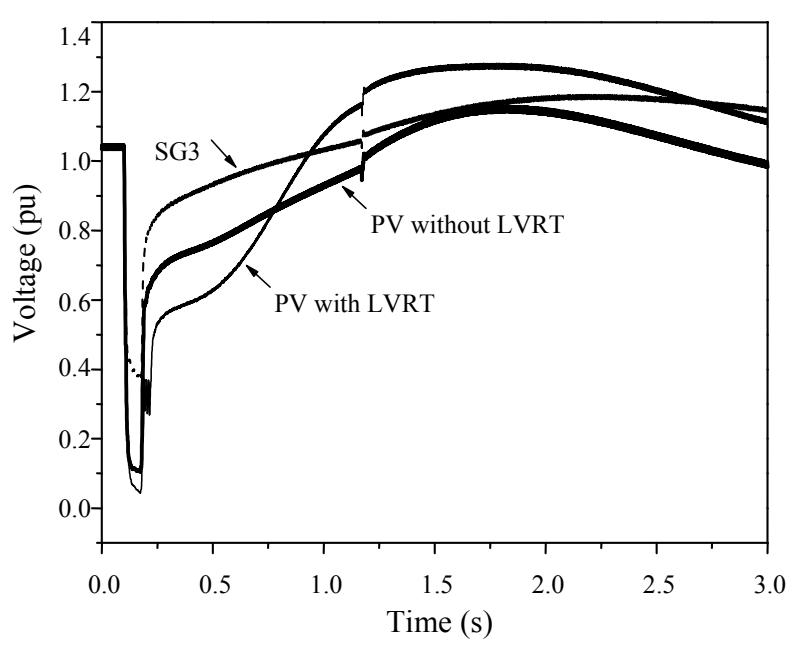

(c) PV or SG3

Fig. 11 Terminal voltages of SG1, SG2 and PV or SG3 (without infinite bus).

\section{Conclusions}

This paper presents a case study assessing the impact of high-penetration $\mathrm{PV}$ on the transient stability of power system. Simulation analyses have been carried out considering existence of LVRT capability of PV inverter and infinite bus in the power system model. Obtained results are summarized as follows:

(1) power system with infinite bus

PV system connected with a relatively large power system or small PV system compared to the power system capacity is assumed. Through the simulation results, it is concluded that the risk of out-of-step of the synchronous generator becomes low in the case that PV without LVRT capability is installed, because the disconnection of PV after the network disturbance increases the power load of the synchronous generator;

(2) power system without infinite bus

PV system connected with a relatively small power system or large PV system compared to the power system capacity is assumed. Through the simulation results, it is concluded that the risk of out-of-step of the synchronous generator becomes high in the case that PV without LVRT capability is installed. This is contrary conclusion with regard to (1). The disconnection of PV due to the voltage sag may lead to out-of-step of synchronous generators in decelerating direction.

Though PV with LVRT capability is installed in the grid system, it can be disconnected from the grid dependent on the condition of the voltage sag and grid fault. It is needless to say that, there is no infinite bus in real power systems. Therefore, it is very important to think about the transient stability and its enhancement for power systems with a huge amount of PV systems installed, for which the results in this paper can be the base.

\section{Acknowledgments}

This work was supported by research grant from 
JSPS (Japan society for the promotion of science)

KAKENHI Grant Number 24656179, 15H03956.

\section{References}

[1] Photovoltaic Power Systems Programme. 2014. Trends 2014 in Photovoltaic Applications. Report IEA-PVPS (International Energy Agency-Photovoltaic Power Systems Programme) T1-25.

[2] Keller, J., and Kroposki, B. 2010. Understanding Fault Characteristics of Inverter-Based Distributed Energy Resources. NREL (National Renewable Energy Laboratory) Technical report, NREL/TP-550-46698.

[3] Du, W., Wang, H. F., and Dunn, R. 2009. "Power System Small-Signal Oscillation Stability as Affected by Large-Scale PV Penetration." Presented at the International Conference on Sustainable Power Generation and Supply, Nanjing, China.
[4] Sakamoto, N., Taniguchi, H., Ota, Y., Nakajima, T., and Chinuki, T. 2012. "Transient Stability Study of One-Machine-to Infinite-Bus Power System under Large Penetration of PV Generation." IEEJ Trans. PE 132 (1): 9-15.

[5] Yagami, M., Hasegawa, T., and Tamura, J. 2012. "Transient Stability Assessment of Synchronous Generator in Power System with High-Penetration Photovoltaics." Journal of Mechanics Engineering and Automation 2 (12): 762-8.

[6] Anderson, P. M., and Fouad, A. A. 2003. Power System Control and Stability. Piscataway: A John Wiley \& Sons, Inc., 38.

[7] Kobayashi, H., and Suzuki, A. 2010. Stable Operation Technique for PCS of PV Power Generation at Grid Recovery after Voltage Sag. System Engineering Research Laboratory, R09015.

[8] IEEJ Technical Committee. 1999. Standard Models of Power System. IEEJ Technical report 754. 